\title{
The prognosis of recurrent low-grade endometrial stromal sarcoma: a retrospective cohort study
}

\author{
Qianwen Dai ${ }^{1}$, Baolin Xu ${ }^{1,2}$, Huanwen $\mathrm{Wu}^{3}$, Yan You ${ }^{3}$, Ming Wu ${ }^{1 *}$ and Lei Li ${ }^{1 *}$ (D)
}

\begin{abstract}
Background: The prognosis of recurrent low-grade endometrial stromal sarcoma (LGESS) is little known. This study was to investigate the survival outcomes of a cohort of patients with recurrent LGESS.

Methods: Patients with primary LGESS diagnosed and treated for first recurrence confirmed by histology in the study center from February 2012 to June 2019 were retrospectively included. The progression-free interval (PFI) after the last treatment for first recurrence and overall survival (OS) since the diagnosis of first recurrence, which were followed up to June 1, 2020, were compared between groups of various therapy modalities.

Results: Fifty-six patients were included, and 43 patients (76.8\%) had definite follow-up outcomes. The 5-year PFI and OS rates were $30.0 \%$ (95\% confidence interval [95\% Cl] 29.2-30.8) and $75.0 \%(68.0-82.0)$, respectively. In univariate analysis, only fertility-sparing treatment, ovarian preservation and surgical treatment had a significant impact on the PFI (hazard ratio [HR] 4.5, 3.1, and 0.2; 95\% Cl 1.5-13.1, 1.3-7.3, and 0.1-0.7; and $p=0.006,0.009$ and 0.006, respectively), but no factor was found to be associated with increased mortality risk. After adjusted with hormone treatment or chemotherapy, surgical treatment had significant effectiveness on OS (HR 0.3 and $0.3,95 \% \mathrm{Cl} 0.1-1.0$ and $0.1-1.0$, $p=0.045$ and 0.049 , respectively). None of the patients with fertility-sparing treatment had successful conception, and all experienced repeated relapse.
\end{abstract}

Conclusion: For patients with recurrent LGESS, fertility-sparing treatment or ovarian preservation should not be provided. Surgery is the treatment of choice, and hormone treatment and/or chemotherapy was effective for the survival benefits of surgical treatment.

Keywords: Low-grade endometrial stromal sarcoma, Fertility-sparing treatment, Ovarian preservation, Surgery, Recurrence, Survival outcomes

\section{Background}

Low-grade endometrial stromal sarcoma (LGESS) is a malignant tumor composed of cells resembling stromal cells of the proliferative-phase endometrium, displaying permeative, infiltrative growth into the myometrium and/

\footnotetext{
*Correspondence: wuming@pumch.cn; lileigh@163.com

1 Department of Obstetrics and Gynecology, Peking Union Medical

College Hospital, Shuaifuyuan No. 1, Dongcheng District, Beijing 100730, China

Full list of author information is available at the end of the article
}

or Iymphovascular spaces [1]. LGESS represents $<1 \%$ of all uterine malignancies but is the second most common uterine malignant mesenchymal tumor with a favorable prognosis $[2,3]$. The five-year disease-specific survival rates are $90 \%$ for stage I or II disease and $50 \%$ for stage III or IV disease $[4,5]$. Due to its indolent course, few studies have reported the prognosis of recurrent LGESS. In a cohort of 42 patients, incomplete surgery and no adjuvant treatment in ESS were associated with poor DFS. Furthermore, resection of recurrent disease is associated with a survival advantage [6], and hormonal treatment original author(s) and the source, provide a link to the Creative Commons licence, and indicate if changes were made. The images or other third party material in this article are included in the article's Creative Commons licence, unless indicated otherwise in a credit line to the material. If material is not included in the article's Creative Commons licence and your intended use is not permitted by statutory regulation or exceeds the permitted use, you will need to obtain permission directly from the copyright holder. To view a copy of this licence, visit http://creativecommons.org/licenses/by/4.0/. The Creative Commons Public Domain Dedication waiver (http://creativeco mmons.org/publicdomain/zero/1.0/) applies to the data made available in this article, unless otherwise stated in a credit line to the data. 
for measurable residual or recurrent low-grade ESS has a high response rate [7]. Because of their efficacy and minimal adverse effects, letrozole $[8,9]$ and medroxyprogesterone $[10,11]$ have been used to treat patients with recurrent or residual LGESS that is difficult to resect surgically. Patients seem to benefit from surgical removal of metastatic lesions, especially pulmonary lesions, followed by progestin therapy [12]. However, these reports had limited sample sizes of patients with recurrent LGESS and restricted effective decision-making with recurrent patients with LGESS.

In this retrospective cohort study, we enrolled all LGESS cases with first recurrence who were diagnosed and treated in the study center to explore the prognostic characteristics and relevant risk factors. The primary objectives were to analyze the progression-free interval (PFI) after the last treatment for first recurrence and overall survival (OS) since the diagnosis of first recurrence. Since fertility-sparing and ovarian preservation surgeries have been shown to be associated with an increased risk of recurrence in LGESS patients [13, 14], we paid special attention to the impact of these issues on the PFI after recurrence.

\section{Methods}

\section{Ethical approval}

The Institutional Review Board from the study center approved this retrospective study (No. SK-1289). All procedures performed in the study involving human participants were in accordance with the ethical standards of the institutional and National Research Committee and with the 1964 Declaration of Helsinki and its later amendments or comparable ethical standards.

\section{Study design}

This was a retrospective cohort study. Patients with primary LGESS diagnosed and treated for first recurrence in the study center from February 2012 to June 2019 were included. Follow-up of fertility and oncologic outcomes was carried out up to June 1,2020 . The primary and recurrent pathological diagnoses were reviewed and confirmed by two pathologists (HW and YY). Patients were excluded if they had an ambiguous or a misdiagnosis of LGESS, had no histological evidence of recurrence, or did not accept surgical treatment for the primary disease. Clinicopathological information, including age at diagnosis of recurrence, symptoms at recurrence, and treatment modalities for recurrence, was collected by reviewing case reports. Special attention was given to fertility-sparing treatment and ovarian preservation in the treatment of recurrence.

\section{Interventions and measures}

Patients accepted treatment with or without surgical interventions. According to surgical entities, patients were divided into groups with or without fertility-sparing surgery and groups with or without ovarian preservation. In this study, fertility-sparing surgery denotes preservation of the uterus and at least one ovary. In this study, patients with fertility-sparing surgery also had records of regular menstruation after all relevant treatments. Ovarian preservation denotes preservation of at least one ovary with or without an intact uterus. Data on surgical routes and residual lesions from surgical records were also collected from the case and pathology reports. The same attention was given to the chemotherapy regimens and courses, hormone therapy regimens and courses, and radiotherapy.

Oncologic outcomes consisted of PFI after the last treatment for first recurrence and OS since the diagnosis of first recurrence, which were followed up until June 1, 2020. All recurrences were confirmed via histological diagnosis by biopsy and/or repeated surgeries. All deaths were confirmed by certification of death.

\section{Statistics}

Comparisons of continuous variables were conducted with parametric methods if assumptions of a normal distribution were confirmed. Nonnormally distributed variables and categorical data involving specific clinicopathological characteristics were compared between the different groups by using nonparametric tests. Survival curves were generated with the Kaplan-Meier method, and proportional hazards models were used to estimate the hazard ratios (HRs) and 95\% confidence intervals (95\% CIs) to assess the effects of treatment modalities on PFI and OS. Unless otherwise stated, all analyses were performed with a two-sided significance level of 0.05 and conducted with the use of the software Statistical Product and Service Solutions (SPSS) Statistics 20.0 (IBM Corporation, Armonk, NY, USA).

\section{Results}

\section{Patient characteristics}

The flow diagram is presented in Fig. 1. In total, 56 patients with repeated LGESS were included in the study. The baseline characteristics of the patients are summarized in Table 1. The pathological evaluation suggested most patients had mitotic activity $<5$ per 10 high power field (80.4\%), or with positive expression of estrogen receptor (ER) (91.1\%) and progesterone receptor (PR) (89.3\%). The median DFS before recurrence and the median age at diagnosis were 34.7 (range 5-188) months and 43.3 (17-70) years, respectively. Twenty-three 


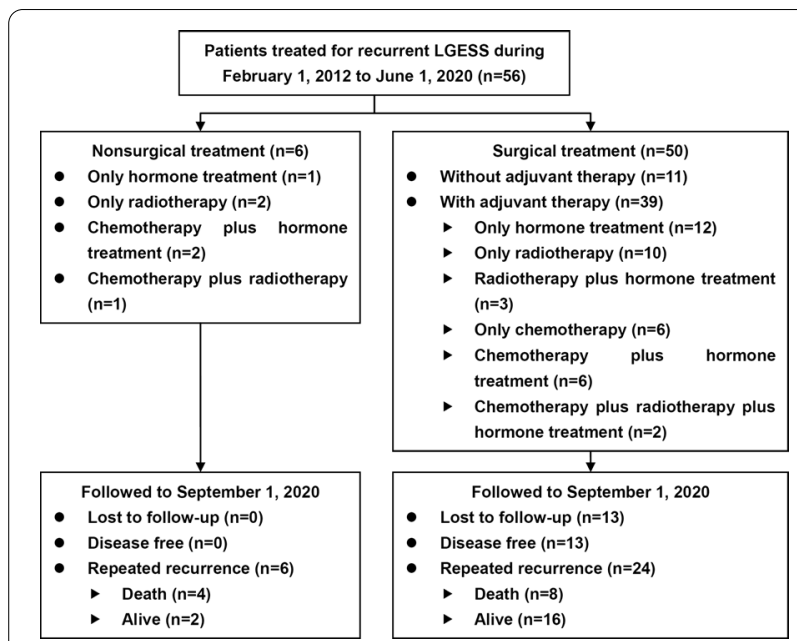

Fig. 1 Flow diagram of the study. LGESS, low-grade endometrial stromal sarcoma

patients $(41.1 \%)$ had metastasis beyond the pelvic cavity or had symptomatic recurrence. Eleven (19.6\%), 44 (78.6\%) and $1(1.8 \%)$ patients were diagnosed by physical examination, imaging evaluation, and accident findings during cesarean section, respectively. Among 44 cases diagnosed by imaging methods, sonography, computed tomography, magnetic resonance imaging and positron emission tomography were primarily performed in 22 (50.0\%), 12 (27.3\%), 6 (13.6\%) and 4 (9.1\%) patients, respectively. Among 23 patients with symptomatic recurrence, abdominal pain or a mass was the most common complaint (12 patients [52.2\%]), followed by vaginal bleeding or a mass $(6[26.1 \%])$ and backache (3 [13.0\%]). The other two patients complained of ileus and severe fatigue.

After recurrence, six patients refused repeated surgery and accepted only GnRHa treatment (1 case), only radiotherapy ( 2 cases), only chemotherapy (1 case), or medroxyprogesterone plus chemotherapy (2 cases). Among the 50 patients who underwent repeated surgeries, 3 had residual lesions. Among 16 and 32 patients with fertility-sparing surgery and ovarian preservation at first treatment, 5 and 6 accepted repeated fertility-sparing surgery and ovarian preservation treatment, respectively. Chemotherapy, radiotherapy and hormone treatment

Table 1 Epidemiological characteristics, treatment and follow-up of enrolled patients

\begin{tabular}{|c|c|}
\hline & Values \\
\hline Extrauterine LGESS, n (\%) & $9(16.1)$ \\
\hline Mitotic activity < 5 per 10 high power field, $n(\%)$ & $45(80.4)$ \\
\hline \multicolumn{2}{|l|}{ Immunohistochemical staining, $\mathrm{n}(\%)$} \\
\hline Positive estrogen receptor & $51(91.1)$ \\
\hline Positive progesterone receptor & $50(89.3)$ \\
\hline DFS after first treatment (months), median (range) & $34.7(5-188)$ \\
\hline Ages at diagnosis of recurrence (years), median (range) & $43.3(17-70)$ \\
\hline Extrapelvic recurrence, $\mathrm{n}(\%)$ & $23(41.1)$ \\
\hline Symptomatic recurrence, n (\%) & $23(41.1)$ \\
\hline Repeated surgeries for first recurrence, $n(\%)$ & $50(89.3)$ \\
\hline With residual lesions, $\mathrm{n} / \mathrm{n}(\%)$ & $3 / 50(6.0)$ \\
\hline Chemotherapy for recurrence, $\mathrm{n}(\%)$ & $17(30.4)$ \\
\hline Radiotherapy for recurrence, $\mathrm{n}(\%)$ & $18(32.1)$ \\
\hline Hormone treatment for recurrence, $\mathrm{n}(\%)$ & $26(46.4)$ \\
\hline \multicolumn{2}{|l|}{ Fertility sparing } \\
\hline Fertility sparing after first treatment, n (\%) & $9(16.1)$ \\
\hline Fertility sparing after first recurrence, $n(\%)$ & $6(10.7)$ \\
\hline \multicolumn{2}{|l|}{ Ovarian preservation } \\
\hline Ovarian preservation after first treatment, $\mathrm{n}(\%)$ & $35(62.5)$ \\
\hline Ovarian preservation after first recurrence, $\mathrm{n}(\%)$ & $9(16.1)$ \\
\hline Loss to follow-up, n (\%) & $13(23.2)$ \\
\hline Repeated recurrence, $\mathrm{n} / \mathrm{n}(\%)$ & $30 / 43(69.8)$ \\
\hline PFI after last treatment for first recurrence (months), median (range) & $18.3(1-121)$ \\
\hline Death, n/n (\%) & $12 / 43(27.9)$ \\
\hline OS after diagnosis of first recurrence (months), median (range) & $43.6(3-349)$ \\
\hline
\end{tabular}

DFS, disease-free survival; PFI, progression-free interval; LGESS, low-grade endometrial stromal sarcoma 
were utilized by 17 (30.4\%), 18 (32.1\%) and 26 (46.4\%) patients, respectively.

\section{Survival outcomes}

Up to June 1, 2020, 13 patients (23.2\%) were lost to follow-up after they finished treatment for recurrence. Among the remaining 43 patients, 30 (69.8\%) experienced repeated recurrence, and $12(27.9 \%)$ patients died, with a median PFI and OS of 18.3 (range 1-121) and 43.6 (3-349) months, respectively. The 5-year PFI and OS rates were $30.0 \%$ (95\% CI $29.2-30.8)$ and $75 \%(68-82)$, respectively, and the 10 -year OS rate was $36 \%(16-56)$.

As shown in Fig. 2, in the Kaplan-Meier analysis, fertility-sparing surgery, ovarian preservation and surgical treatment had a significant impact on the PFI after the last treatment for first recurrence (HR 4.5, 3.1, and 0.2, 95\% CI 1.5-13.1, 1.3-7.3, and 0.1-0.7, $p=0.006,0.009$ and 0.006, respectively). The median PFIs of patients with and without fertility-sparing surgery were 8 (range $3-18)$ and 29 (1-121) months, the median PFIs with and without ovarian preservation were $8(2-57)$ and 25 (1-121) months, and the median PFIs with surgical and nonsurgical treatment were $25(1-121)$ and $6(2-37)$ months, respectively. As shown in Fig. 3, after adjusted by repeated surgeries, fertility-sparing treatment still resulted in a higher risk of recurrence (HR 3.8, 95\% CI 1.3-11.5, $p=0.016$ ), but ovarian preservation had no significant impact on OS (HR 2.2, 95\% CI 0.8-5.9, $p=0.107)$. Of the three patients with residual lesions after surgery, one was lost to follow-up, one died of disease progression 14 months after the diagnosis of recurrence, and one had disease progression 8 months after the last treatment but remained alive during the study period.

The median OS times of the patients with and without fertility-sparing surgery were 59 (range 8-101) and 44
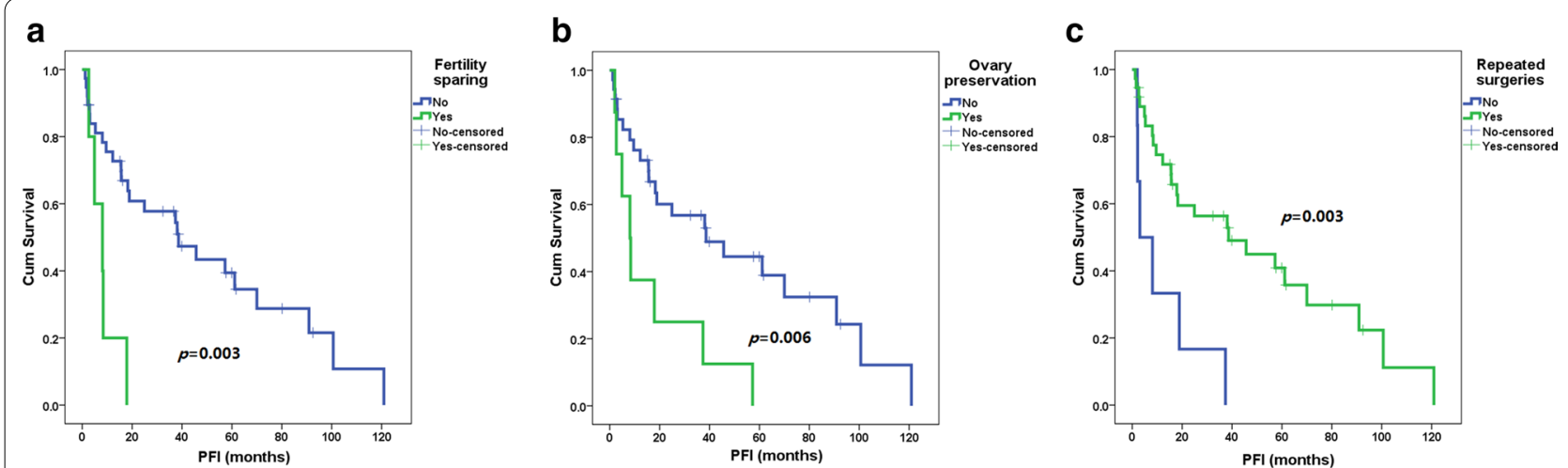

Fig. 2 Progression-free interval (PFI) after first recurrence of patients with and without fertility-sparing surgery (a), or with and without ovarian preservation (b), or with and without repeated surgeries for recurrence (c) by Kaplan-Meier analysis
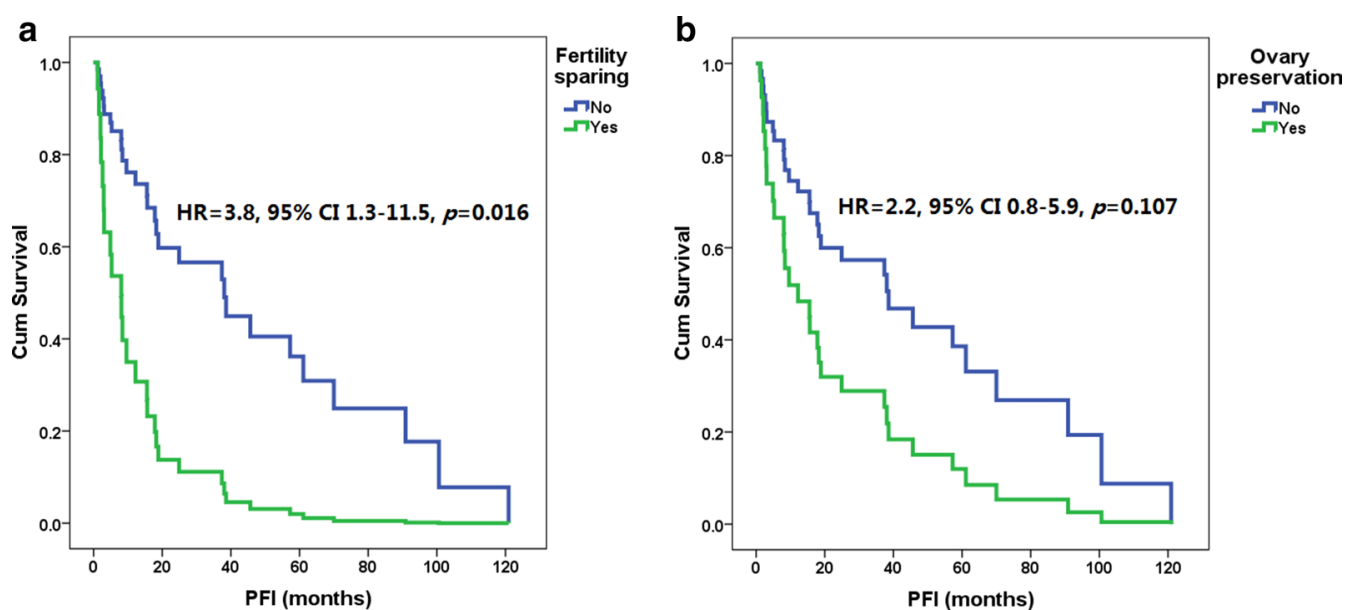

Fig. 3 Progression-free interval (PFI) in patients with and without fertility-sparing surgery (a) and in patients with and without ovarian preservation (b). 95\% Cl 95\% confidence interval, HR hazard ratio 
(3-349) months, the median OS with and without ovarian preservation were 69 (8-349) and 44 (3-229) months, and the median OS times with surgical and nonsurgical treatment were 44 (3-229) and 31 (12-349) months, respectively. No factor, including fertility-sparing surgery (Fig. 2c, HR 1.0 [95\% CI 0.1-7.7], $p=0.977$ ), ovarian preservation (Fig. 2d, HR 0.3 [95\% CI 0.04-2.5], $p=0.276)$, and surgical treatment (HR $0.3 \quad\left[\begin{array}{lll}9 & \mathrm{CI}\end{array}\right.$ $0.1-1.0], p=0.052)$, was found to be associated with increased mortality risk. However, as shown in Fig. 4, after adjusted with hormone treatment or chemotherapy, surgical treatment had significant impact on OS (HR 0.3 and 0.3 [95\% CI $0.1-1.0$ and $0.1-1.0$ ], $p=0.045$ and 0.049 , respectively). After adjusted with radiotherapy, surgical treatment still had no significant impact on OS (HR 0.3 [95\% CI 0.1-1.1], $p=0.066$ ).

Regarding repeated recurrent sites, in 11 and 19 patients with recurrence beyond and within the pelvic cavity at first recurrence, 6 (54.5\%) and 7 (36.8\%) developed new recurrence beyond the pelvic cavity, respectively $(p=0.287)$. As shown in the Additional file 1, 11 patients underwent retroperitoneal lymphadenectomy at first treatment and had no recurrences in the retroperitoneal cavity. Among 45 patients without retroperitoneal lymphadenectomy at first treatment, 4 of $45(8.9 \%)$ and 2 of $22(9.1 \%)$ patients had recurrences in the retroperitoneal lymph nodes at first and repeated recurrence, respectively. However, only one case had recurrence solely in the retroperitoneal cavity at first recurrence. In other cases, retroperitoneal recurrences were accompanied by recurrences in other sites.

Pathological characteristics of ER, PR expression and low mitotic activity were not associated with PFI (logrank $p$ values $0.495,0.119$ and 0.564 ) or OS (log-rank $p$ values $0.473,0.421$ and 0.070 ) in the Kaplan-Meier analysis.

Hormone treatment was not associated with PFI or OS in Kaplan-Meier analysis (log-rank $p$ values 0.714 and 0.717 ), or adjusted with ER status ( $p$ values 0.806 and 0.651 ), or PR status ( $p$ values 0.868 and 0.595 ), or surgical treatment (HR 0.9 and 0.7 [95\% CI 0.4-2.1 and $0.2-2.4$ ], $p$ values 0.854 and 0.553 ).

Chemotherapy was not associated with PFI or OS in Kaplan-Meier analysis (log-rank $p$ values 0.534 and 0.647 ), or adjusted with surgical treatment (HR 1.0 and

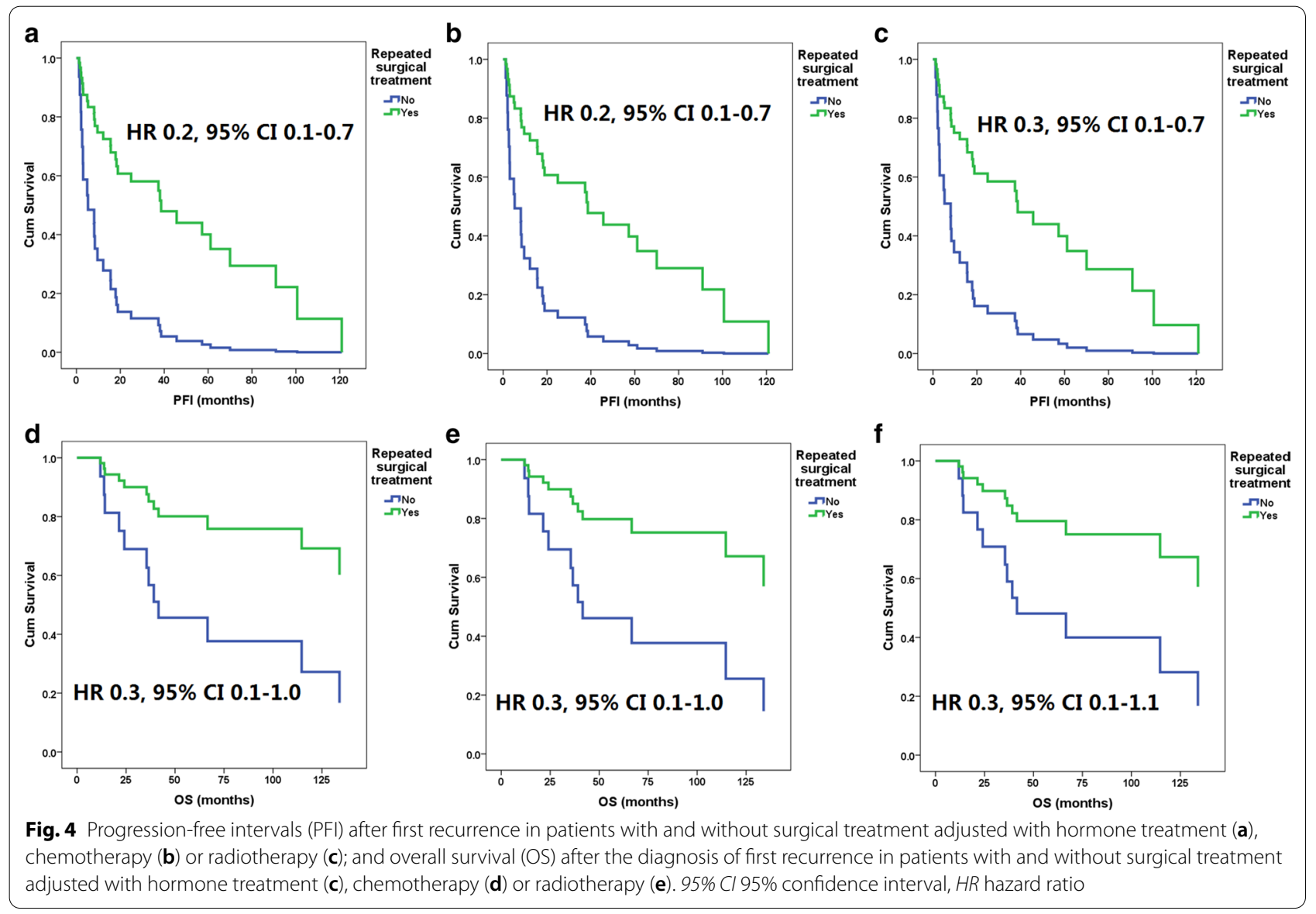


0.7 [95\% CI $0.5-2.2$ and $0.2-2.4$ ], $p$ values 0.806 and $0.584)$.

\section{Fertility outcomes}

Among six patients requesting repeated fertility-sparing treatment, one was lost to follow-up, and the other five experienced repeated recurrence. One patient (LGE0004 in the Additional file 1) was diagnosed with a first recurrence during her cesarean section for her second live birth, when metastasis to the retroperitoneal lymph nodes was noticed. Then, she underwent repeated fertility-sparing surgeries again (resection of metastatic lymph nodes) without adjuvant therapy. However, five months after surgery, recurrence in the uterus was noticed; she accepted non-fertility-sparing surgeries and remained alive up to the end of the follow-up.

\section{Discussion}

We report the prognosis of recurrent LGESS in a cohort of 56 patients. To our knowledge, this is the largest population to date. Compared with primary LGESS patients who had 5-year DFS and OS rates of over 90\% 5-year, patients with LGESS recurrence had higher recurrence and mortality rates. Patients with fertility-sparing treatment, ovarian preservation, or a treatment modality other than repeated surgeries all had significantly higher recurrence rates. Although no factor was found to be associated with mortality risk, surgeries seemed to have marginal protective effects on OS. These findings provide a substantial basis for decision-making in the selection of treatment for LGESS recurrence.

Fertility-sparing treatment has been reported only in case reports for primary uterine LGESS [15-23], which lack evidence of safety and effectiveness. The safety of fertility-sparing treatment could be indirectly investigated by reviewing evidence of ovarian preservation. Ovarian preservation has been proven to increase the risk of LGESS recurrence in retrospective cohort studies [24-26] and in a systematic review [14]. Estrogens or tamoxifen treatment also increase the risk of recurrence [27-29], while menopause is a protective factor associated with improved PFS among patients with LGESS [30]. In addition, numerous reports have revealed the role of endometriosis in the pathogenesis of extrauterine LGESS $[31,32]$. These findings all show the high risk of recurrence associated with ovarian preservation and, similarly, the high risk associated with fertility-sparing treatment. In our current study, all patients undergoing repeated fertility-sparing surgeries experienced repeated recurrence without successful conception. Based on these findings, we concluded that patients with recurrent LGESS who undergo fertility-sparing treatment have a high risk recurrence, but with few chances of conception; oophorectomy should be incorporated into the set of repeated surgeries for all recurrent LGESS patients, if applicable.

In our study, surgical treatment led to a better PFI and, possibly, a better OS for recurrent LGESS patients. Incomplete surgery and no adjuvant treatment in ESS are associated with poor DFS [6]. Several reports have confirmed that tumor size is associated with the survival outcomes of LGESS patients [5, 33, 34]. Even for patients with tumors that are difficult to resect surgically, if surgical treatment can minimize the tumor volume, sustained treatment may achieve favorable disease control [7-11]. Therefore, the principle of efforts to perform complete surgeries should also be utilized in recurrent LGESS, and surgery should be the choice of treatment for recurrent LGESS. Due to the scarcity of current evidence, a largesample study is needed to determine the role of combination therapy consisting of surgeries and other modalities.

In our study, for patients without retroperitoneal lymphadenectomy at first treatment, approximately $10 \%$ developed retroperitoneal recurrences. The role of lymphadenectomy in LGESS is controversial. There is no sufficient indication for a systematic LND for patients with early-stage LGESS $[35,36]$. A systematic LND might be necessary if enlarged lymph nodes are detected by image graphology or observation during surgery [37, 38]. In addition, lymph node metastasis does not seem to affect the excellent OS of LGESS patients [39]. Even though a high proportion $(10 \%)$ of recurrent patients had recurrence in the retroperitoneal space in our study, only one patient had disease restricted in this location. These findings suggest that systematic lymphadenectomy may be eliminated in the first surgical treatment for LGESS.

In our study, we discovered that estrogen and progesterone receptor expression or mitotic activities had no significant impact on the survival outcomes of recurrent LGESS patients. There was no study on the association of mitotic activity with survival outcomes in recurrent LGESS. Low mitotic activities were associated with decreased risk of recurrence or mortality in primary diseases by some reports [25, 40, 41], but the limited sample sizes restrict the generation of their findings. The status of hormone receptors provided indication of utilization of hormone treatment. However, even for primary tumors, the effects of hormone treatment have lots of debates [30, 42-45]. In our study, although hormone treatment had no significant impact on the survival outcomes, it seemed to be able to provide survival benefits together with surgical treatment for recurrent patients.

In our study, just similar to hormone treatment, chemotherapy had no significant impact on survival outcomes, but could benefit the patients with surgical treatment. Administration of adjuvant radiotherapy 
or chemotherapy is not routinely used in LGESS and its role is still debated [35, 45]. Retrospective cohort study revealed that chemotherapy had no significant effect on disease-free survival of LGESS [2]. However, in the nomogram for survival of LG-ESS, chemotherapy was included as a variable in population-based analysis [33]. For the recurrent LGESS, to our knowledge, we first discovered the effectiveness of adjuvant chemotherapy for surgical treatment.

The relatively large sample size and detailed description of treatment are the strengths of our study. However, the retrospective study design is the main limitation of our study. A high rate of loss to follow-up (23.2\%) after complete treatment for recurrence is another important limitation, as this restricted the generalization of our findings. National or international registry systems would provide a high-quality platform for the comprehensive analysis of rare diseases, such as recurrent LGESS.

\section{Conclusions}

Fertility-sparing surgery or ovarian preservation treatment resulted in an inferior PFI without successful conception, and should not be offered to patients with recurrent LGESS. Surgical treatment was associated with better survival outcomes than other therapy modalities without surgeries, especially followed by adjuvant therapy of hormone treatment and/or chemotherapy, and should be the choice for recurrent LGESS. Retroperitoneal involvement occurred in approximately $10 \%$ of patients with first and repeated recurrences but seldom occurred alone, and systematic lymphadenectomy seemed to unnecessary in the primary treatment for LGESS. Pathological characteristics had no significant impact on survival outcomes.

\section{Abbreviations}

95\% Cl: 95\% Confidence interval; DFS: Disease-free survival; HR: Hazard ratio; LGESS: Low-grade endometrial stromal sarcoma; OS: Overall survival; PFI: Progression-free interval.

\section{Supplementary Information}

The online version contains supplementary material available at https://doi. org/10.1186/s13023-021-01802-8.

Additional file 1. Raw data of this study.

\section{Acknowledgements}

None.

\section{Authors' contributions}

$L L$ and MW conceived of the original idea for the study, interpreted results, carried out the statistical analysis, edited the paper and was overall guarantor. QD obtained ethical approval, contributed to the preparation of the data set, interpreted results and contributed to drafts of the paper. BX contributed to the study design, interpretation of results and commented on drafts of the paper. YY and HW conducted the pathological evaluation. All authors have approved the final version of the manuscript.

Funding

This study is supported by the CAMS Innovation Fund for Medical Sciences (CIFMS-2017-12M-1-002). The funders had no role in the study design, data collection and analysis, decision to publish, or preparation of the manuscript.

Availability of data and materials

All data of this study has been contained in the supplement file.

\section{Declarations}

Ethics approval and registration

The Institutional Review Board of Peking Union Medical College Hospital has approved this study (No. SK-1289).

\section{Consent for publication}

Consents for publication have been obtained from all patients.

\section{Author details}

${ }_{1}^{1}$ Department of Obstetrics and Gynecology, Peking Union Medical College Hospital, Shuaifuyuan No. 1, Dongcheng District, Beijing 100730, China. ${ }^{2}$ Department of Obstetrics and Gynecology, the Second People's Hospital of Jingdezhen, Jingdezhen 333099, China. ${ }^{3}$ Department of Pathology, Peking Union Medical College Hospital, Beijing 100730, China.

Received: 19 November 2020 Accepted: 30 March 2021

Published online: 07 April 2021

\section{References}

1. Kurman RJ, Carcangiu ML, Herrington CS, Young RH, editors. WHO classification of tumours of female reproductive organs. 4th ed. International Agency for Research on Cancer (IARC): Lyon; 2014.

2. Zhou J, Zheng H, Wu SG, He ZY, Li FY, Su GQ, et al. Influence of different treatment modalities on survival of patients with low-grade endometrial stromal sarcoma: a retrospective cohort study. Int J Surg. 2015;23:147-51.

3. Trope CG, Abeler VM, Kristensen GB. Diagnosis and treatment of sarcoma of the uterus: a review. Acta Oncol. 2012;51:694-705.

4. Abeler VM, Royne O, Thoresen S, Danielsen HE, Nesland JM, Kristensen GB. Uterine sarcomas in Norway. A histopathological and prognostic survey of a total population from 1970 to 2000 including 419 patients. Histopathology. 2009;54:355-64.

5. Seagle BL, Shilpi A, Buchanan S, Goodman C, Shahabi S. Low-grade and high-grade endometrial stromal sarcoma: a National Cancer Database study. Gynecol Oncol. 2017;146:254-62.

6. Agarwal R, Rajanbabu A, Nair IR, Satish C, Jose G, Unikrishnan UG. Endometrial stromal sarcoma-A retropsective analysis of factors affecting recurrence. Eur J Obstet Gynecol Reprod Biol. 2017;216:92-7.

7. Dahhan T, Fons G, Buist MR, Ten Kate FJ, van der Velden J. The efficacy of hormonal treatment for residual or recurrent low-grade endometrial stromal sarcoma: a retrospective study. Eur J Obstet Gynecol Reprod Biol. 2009;144:80-4.

8. Yamaguchi M, Erdenebaatar C, Saito F, Motohara T, Miyahara Y, Tashiro H, et al. Long-term outcome of aromatase inhibitor therapy with letrozole in patients with advanced low-grade endometrial stromal sarcoma. Int J Gynecol Cancer. 2015;25:1645-51.

9. Ryu H, Choi YS, Song IC, Yun HJ, Jo DY, Kim S, et al. Long-term treatment of residual or recurrent low-grade endometrial stromal sarcoma with aromatase inhibitors: a report of two cases and a review of the literature. Oncol Lett. 2015;10:3310-4.

10. Mizuno M, Yatabe Y, Nawa A, Nakanishi T. Long-term medroxyprogesterone acetate therapy for low-grade endometrial stromal sarcoma. Int J Clin Oncol. 2012;17:348-54.

11. Nakayama K, Ishikawa M, Nagai Y, Yaegashi N, Aoki Y, Miyazaki K. Prolonged long-term survival of low-grade endometrial stromal sarcoma patients with lung metastasis following treatment with medroxyprogesterone acetate. Int J Clin Oncol. 2010;15:179-83. 
12. Garavaglia E, Pella F, Montoli S, Voci C, Taccagni G, Mangili G. Treatment of recurrent or metastatic low-grade endometrial stromal sarcoma: three case reports. Int J Gynecol Cancer. 2010;20:1197-200.

13. Bai $H$, Yang J, Cao D, Huang $H$, Xiang $Y$, Wu M, et al. Ovary and uterus-sparing procedures for low-grade endometrial stromal sarcoma: a retrospective study of 153 cases. Gynecol Oncol. 2014;132:654-60.

14. Nasioudis D, Ko EM, Kolovos G, Vagios S, Kalliouris D, Giuntoli RL. Ovarian preservation for low-grade endometrial stromal sarcoma: a systematic review of the literature and meta-analysis. Int J Gynecol Cancer. 2019;29:126-32

15. Maeda $O$, Moritani S, Ichihara S, Inoue T, Ishihara $Y$, Yamamoto S, et al. Long-term survival in low-grade endometrial stromal sarcoma with childbirth and multidisciplinary treatment: a case report. J Med Case Rep. 2015;9:233.

16. Laurelli G, Falcone F, Scaffa C, Messalli EM, Del Giudice M, Losito S, et al. Fertility-sparing management of low-grade endometrial stromal sarcoma: analysis of an institutional series and review of the literature. Eur J Obstet Gynecol Reprod Biol. 2015;195:61-6.

17. Jin Y, Li Y, Deng CY, Tian QJ, Chen H, Pan LY. Fertility-sparing treatment of low-grade endometrial stromal sarcoma. Int J Clin Exp Med. 2015;8:5818-21.

18. Zhan R, Wen H, Gao X, Yin L. Successful term pregnancy after laparoscopic surgery of low grade endometrial stromal sarcoma. Chin Med (Engl). 2014;127:391-2.

19. Dong R, Pang Y, Mao H, Yang N, Liu P. Successful pregnancy following conservative management of low-grade endometrial stromal sarcoma: a case report. Oncol Lett. 2014;7:1039-42.

20. Choi MC, Kim G, Hwang YY. Fertility-sparing management combined with photodynamic therapy for endometrial stromal sarcoma: a case report. Photodiagn Photodyn Ther. 2014;11:533-6.

21. Delaney AA, Gubbels AL, Remmenga S, Tomich P, Molpus K. Successful pregnancy after fertility-sparing local resection and uterine reconstruction for low-grade endometrial stromal sarcoma. Obstet Gynecol. 2012;120:486-9.

22. Koskas M, Morice P, Yazbeck C, Duvillard P, Walker F, Madelenat P. Conservative management of low-grade endometrial stromal sarcoma followed by pregnancy and severe recurrence. Anticancer Res. 2009;29:4147-50.

23. Stadsvold JL, Molpus KL, Baker JJ, Michael K, Remmenga SW. Conservative management of a myxoid endometrial stromal sarcoma in a 16-yearold nulliparous woman. Gynecol Oncol. 2005;99:243-5.

24. Feng W, Hua K, Malpica A, Zhou X, Baak JP. Stages I to II WHO 2003-defined low-grade endometrial stromal sarcoma: how much primary therapy is needed and how little is enough? Int J Gynecol Cancer. 2013;23:488-93.

25. Feng W, Hua K, Gudlaugsson E, Yu Y, Zhou X, Baak JP. Prognostic indicators in WHO 2003 low-grade endometrial stromal sarcoma. Histopathology. 2013;62:675-87.

26. Li N, Wu LY, Zhang HT, An JS, Li XG, Ma SK. Treatment options in stage I endometrial stromal sarcoma: a retrospective analysis of 53 cases. Gynecol Oncol. 2008;108:306-11.

27. Pink D, Lindner T, Mrozek A, Kretzschmar A, Thuss-Patience PC, Dörken $B$, et al. Harm or benefit of hormonal treatment in metastatic low-grade endometrial stromal sarcoma: single center experience with 10 cases and review of the literature. Gynecol Oncol. 2006;101:464-9.

28. Kashiyama T, Oda K, Kawana K, Arimoto T, Kanetaka Y, Takazawa Y, et al. Low-grade endometrial stromal sarcoma developing in a postmenopausal woman under toremifene treatment for breast cancer. J Obstet Gynaecol Res. 2013;39:424-9.
29. Liao JB, Lin JY. Estrogen receptor expression in an endometrial stromal sarcoma after tamoxifen therapy. Eur J Gynaecol Oncol. 2001;22:417-9.

30. Zhang Y, Li Y, Huang H, Yang J, Wu M, Jin Y, et al. Low-grade endometria stromal sarcoma and uterine adenosarcoma: a comparison of clinical manifestations and outcomes. J Cancer. 2019;10:3352-60.

31. Oliva E, Egger JF, Young RH. Primary endometrioid stromal sarcoma of the ovary: a clinicopathologic study of 27 cases with morphologic and behavioral features similar to those of uterine low-grade endometrial stromal sarcoma. Am J Surg Pathol. 2014;38:305-15.

32. Kondi-Paphitis A, Smyrniotis B, Liapis A, Kontoyanni A, Deligeorgi H. Stromal sarcoma arising on endometriosis: a clinicopathological and immunohistochemical study of 4 cases. Eur J Gynaecol Oncol. 1998;19:588-90.

33. Wu J, Zhang H, Li L, Hu M, Chen L, Xu B, et al. A nomogram for predicting overall survival in patients with low-grade endometrial stromal sarcoma: a population-based analysis. Cancer Commun (Lond). 2020;40:301-12.

34. Barney B, Tward JD, Skidmore T, Gaffney DK. Does radiotherapy or lymphadenectomy improve survival in endometrial stromal sarcoma? Int J Gynecol Cancer. 2009;19:1232-8.

35. Capozzi VA, Monfardini L, Ceni V, Cianciolo A, Butera D, Gaiano M, et al. Endometrial stromal sarcoma: a review of rare mesenchymal uterine neoplasm. J Obstet Gynaecol Res. 2020;46:2221-36.

36. Wang M, Meng SH, Li B, He Y, Wu YM. Survival outcomes of different treatment modalities in patients with low-grade endometrial stromal sarcoma. Chin Med J (Engl). 2019;132:1128-32.

37. Zhang Y, Li N, Wang W, Yao H, An J, Sun Y, et al. Long-term impact of lymphadenectomies in patients with low-grade, early-stage uterine endometrial stroma sarcoma. J Obstet Gynaecol Res. 2020;46:654-62.

38. Signorelli M, Fruscio R, Dell'Anna T, Buda A, Giuliani D, Ceppi L, et al. Lymphadenectomy in uterine low-grade endometrial stromal sarcoma: an analysis of 19 cases and a literature review. Int J Gynecol Cancer. 2010;20:1363-6.

39. Shah JP, Bryant CS, Kumar S, Ali-Fehmi R, Malone JM Jr, Morris RT. Lymphadenectomy and ovarian preservation in low-grade endometrial stromal sarcoma. Obstet Gynecol. 2008;112:1102-8.

40. Bodner K, Bodner-Adler B, Obermair A, Windbichler G, Petru E, Mayerhofer $S$, et al. Prognostic parameters in endometrial stromal sarcoma: a clinicopathologic study in 31 patients. Gynecol Oncol. 2001;81:160-5.

41. Feng W, Malpica A, Skaland I, Gudlaugsson E, Robboy SJ, Dalen I, et al. Can proliferation biomarkers reliably predict recurrence in World Health Organization 2003 defined endometrial stromal sarcoma, low grade? PLOS ONE. 2013:8:e75899.

42. Zang Y, Dong M, Zhang K, Gao C, Guo F, Wang Y, et al. Hormonal therapy in uterine sarcomas. Cancer Med. 2019;8:1339-49.

43. Cui R, Cao G, Bai H, Zhang Z. The clinical benefits of hormonal treatment for LG-ESS: a meta-analysis. Arch Gynecol Obstet. 2019;300:1167-75.

44. Stewart LE, Beck TL, Giannakopoulos NV, Rendi MH, Isacson C, Goff BA. Impact of oophorectomy and hormone suppression in low grade endometrial stromal sarcoma: a multicenter review. Gynecol Oncol. 2018;149:297-300.

45. Burghaus S, Halmen S, Gass P, Mehlhorn G, Schrauder MG, Lux MP, et al. Outcome and prognosis in uterine sarcoma and malignant mixed Mullerian tumor. Arch Gynecol Obstet. 2016;294:343-51.

\section{Publisher's Note}

Springer Nature remains neutral with regard to jurisdictional claims in published maps and institutional affiliations. 